\title{
SUGGESTIONS TOWARDS A SIMPLIFIED SYSTEM OF WEATHER SIGNALS, TERMED THE INDEX WEATHER SIGNAL SYSTEM.
}

By Joshua Pusey.

[Presented at the Stated Meeting of the FRAnkLin Institute, held June i6, I886.]

In the effort to learn and to recollect the well-known weather signals now in use, consisting of the red and blue suns, stars and crescents, I was struck, as others have doubtless been, with the difficulty of fixing and retaining the same in memory because of the confusion of arbitrary colors and forms. It occurred to me that a system might be devised which would possess the salient advantages of being simpler, independent of essential color and form, readily learned and recollected, and more economical than the system now in vogue.

My reflections upon this subject led me to devise the system which I shall now proceed to describe, premising, however, that, although it is not claimed to be perfect, it is thought to be better than that in use.

In this system, movable signal bands are used in connection with a flag, or rotatable pointers pivoted on a common centre, as hereinafter described.

Referring to the drawings, Fig. $I$ represents a flag, $A$, preferably white, having a vertical black stripe or bar $b$ (termed the general rain or snow bar) at one side of the centre. The flag is always hung with this stripe in vertical position. The flag is divided into six (imaginary) fields; three of which are horizontal temperature fields and three vertical weather fields.

The top or higher part is the higher temperature field; the bottom the lower temperature field, and between the two the stationary temperature field. These fields are suggested by the relative position of the mercury in the thermometer, which is higher with higher temperature, etc.

One side or edge of the flag is the local rain or snow field; the other (indicated by the black stripe $b$ ) is the general rain or snow field, and between the two the fair or clear weather field. 


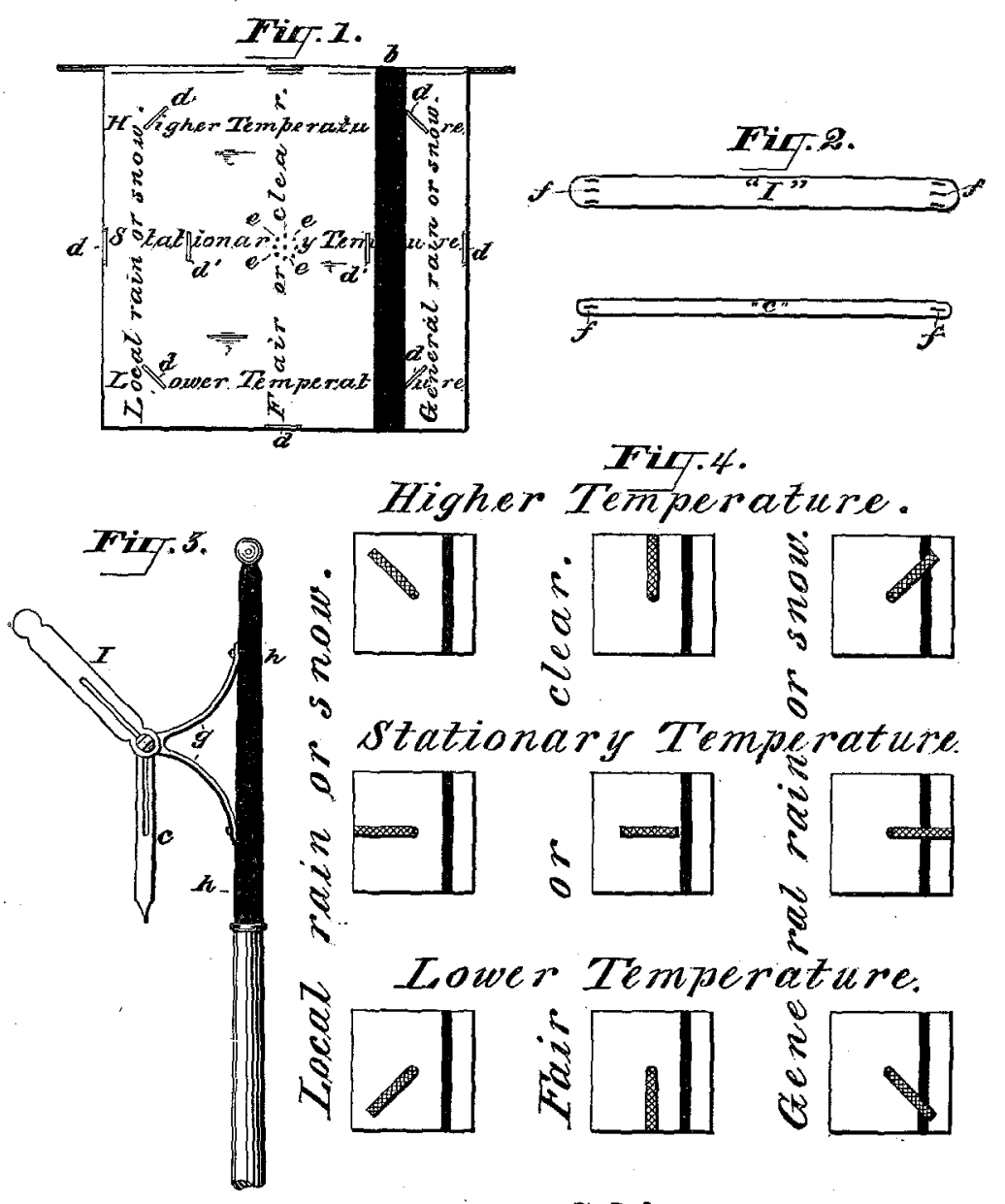

\section{cold-wave.}

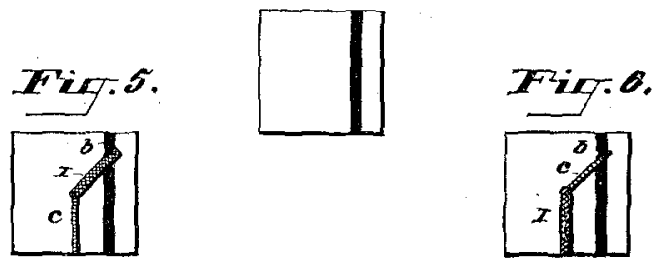

Proposed System of Weather Signals. (Pusey.) 
All that is essential to be learned is the locations of these three temperature and three weather fields, which may be quickly and permanently done.

To indicate the particular signals required-both temperature and weather being indicated at the same time-I employ two bands, Fig. 2, of silk or bunting, one of which, $I$, is broad and is termed the "index," and whose function is to indicate the immediate probabilities; and the other, $c$, relatively narrow and termed the "change" or follower band, whose function is to indicate the weather probabilities following those indicated by the index band. The obvious object of this marked difference in the widths of these bands is to enable the one to be readily distinguished from the other. Their particular color or colors is unimportant, so that they be in strong contrast to that of the flag. Red for the index and blue for the change are preferred.

The flag is provided with button-hole slits $d$, equidistant from the centre, with the single exception of two slits (marked $d^{\prime}$ ), a line connecting which slits traverses the stationary temperature and fair or clear weather fields.

At the centre of the flag, on both sides, are buttons $e$, for attaching the ends of the two bands, $I$ and $c$, which are provided with button-holes $f$, at each end.

The bands are passed through one of slits $d$, according to the signal to be displayed, and the ends respectively are buttoned on both sides of the flag.

For the stationary temperature, clear or fair weather signal, the band or bands pass through the slits $d^{\prime}$, so that the band will be in the two fields at the same time.

When a large flag is used, the bands may be further kept in place by means of light transverse straps at suitable intervals apart, the bands being passed under said straps.

When it is not desirable to use a flag, a device similar to that shown in Fig. 3 may be employed. This consists of a kind of "semaphore," composed of bands or pointers, $I$ and $c$, corresponding to the index and change bands for the flag.

The pointers swing from a pivot $x$ at the junction of the ends of a bracket $g$ that is fixed to and some distance below the top of a pole $h$. The former may be brought to any required position and there held by means of a thumb screw or other device. The 
upper portion of the pole takes the place of the general rain or snow bar $b$ of the flag.

The pointers are provided with longitudinal slots $s$, so as to allow them to be shifted towards the pivot, in order to make the stationary temperature clear or fair weather signal.

In order that the signals may be seen from any direction, there may be two sets of pointers connected with the pole in planes at right angles to each other.

Frequently, and in fact generally, the change signals are not used, in which case the narrow or change pointer may be turned back of the broad one and thereby be concealed from view. The pointers are removed for the cold-wave signal, and the flag is left bare for the same signal.

The diagrams, Fig. 4, show all the various signals. These correspond with those with which the general public is familiar. Cautionary or other signals may be made, if desired, by adopting arbitrary or, perhaps, suggestive shapes or colors for the pointers or bands.

To illustrate the difference between this system and the one now in use, the signal "higher temperature, local rain or snow," at the top of Fig. 4, on the left side, is made in the latter system by means of a flag with a red sun and one with a blue star.

The signal, Fig. 5, "higher temperature, general rain or snow, followed by lower temperature, fair or clear weather," is indicated in the present system by four flags in succession, to wit, a red ball, a blue ball, a red crescent and a blue crescent.

The signal, Fig. 6, "lower temperature, fair or clear weather, followed by higher temperature, general rain or snow," is indicated in the latter system by a red crescent, a blue crescent, a red ball and a blue ball.

It will be observed that the broad, or " index," band or pointer is always read first.

It may also be remarked that the signals in the proposed system form a straight Jine diagram; that is to say, the pointers or bands with the black stripe, or general rain bar. Thus a person glancing at the flag, or semaphore, will have the particular signal diagram impressed upon his mind, and will be able to carry it in memory for the day; whilst it is extremely difficult 
to recollect the suns, stars and crescents, and their colors and order of succession, when several signals are displayed.

The salient advantages of the proposed system may be summed up as follows :

(I.) It is simple and suggestive, and readily learned and fixed in the mind, because of a certain easy association of ideas.

(2.) There is no confusion of colors and forms.

(3.) The signals being indicated by direction, they may be distinguished at long distances, far beyond those at which the colors of the suns, stars, etc., of the present system and their forms can be made out, unless of comparatively large dimensions.

This is especially the case with the semaphore construction, as the direction of the bands, or pointers, can be seen with the naked eye a distance of several miles.

(4.) It is much more economical, the cost of the one flag with bands, or the semaphore, being but a fraction of that of the flags required in the system now used.

\section{HERSCHEL vs. JEVONS ET AL.}

\section{By Pliny Earle Chase, LL. D.}

In the Journal OF. THE FrankLIN INSTITUTE, for January, I 885 , (p. 39), I pointed out "An Error by Maxwell," arising from a simple transposition of figures, a mistake with which all book-keepers are familiar. The error has led many recent writers, in popular text books and scientific journals, to assign to the luminiferous xther a density seventy-five per cent. greater than is justified by Maxwell's hypothesis.

An error of much greater importance has arisen from a misinterpretation of some passages in Herschel's "Lectures on Light." Jevons says (Principles of Science, ii, 145), "Sir John Herschel has calculated the amount of force which may be supposed, according to the undulatory theory of light, to be exerted at each point of space, and finds it to be I,I48,000,000,000 times the elastic force of ordinary air at the earth's surface, so that the pressure of the ather upon a square inch of surface must be about I 7,000,000,000,000, or seventeen billions of pounds; yet we live and move, without appreciable resistance, through this medium Whole No. Vol. CXXII.-(Third Series. Vol. xcii.) 